\title{
A New Astrophysical Constraint on Radiatively Decaying Neutrinos
}

\author{
Shiv K. Sethi \\ Inter University Centre for Astronomy and Astrophysics \\ Post Bag 4, Ganeshkhind, \\ Pune 411007
}

\begin{abstract}
We calculate constraints on radiatively decaying neutrinos from the recent detection of singly ionized helium in the diffuse intergalactic medium (IGM) at $z \simeq 3.3$. We consider a model in which neutrinos predominantly decay into invisible relativistic particles with a rate $\tau^{-1}$, and with a small branching ratio into the radiative mode. To satisfy the observation of singly ionized helium, which puts a lower bound on the number density of singly ionized helium in the IGM, we show that: for $\tau \gtrsim 10^{18} \sec \left(1 \mathrm{eV} / m_{\nu}^{2}\right)$, transition moment of neutrinos $\mu_{12}$ is constrained to be $\leq 4-8 \times 10^{-17} \mu_{B}$ for $110 \mathrm{eV} \lesssim m_{\nu} \lesssim 10 \mathrm{keV}$. We compare this bound with other astrophysical and cosmological bounds on radiatively decaying neutrinos.
\end{abstract}

PACS numbers: $98.60 . \mathrm{Hj}, 98.80 . \mathrm{Cq}, 98.70 . \mathrm{Vc}, 98.80 . \mathrm{bq}$ 
Radiatively decaying neutrinos have found widespread application in cosmology and astrophysics [13]. On the other hand, a formidable array of constraints exist on radiative lifetime of radiatively decaying neutrinos, from studies of various disparate phenomena in cosmology and astrophysics [3-8]. In this paper, we point out another constraint which comes from the existence of primordial elements (hydrogen and helium) in the diffuse IGM at high redshifts. The search of primordial elements in the diffuse IGM is crucial to understanding the universe at early epochs (Gunn-Peterson (GP) test [9]). So far there is no definite evidence of the presence of either neutral hydrogen or neutral helium in diffuse IGM [10,11]. Last year, first detection of singly ionized helium in diffuse IGM was reported by Jakobsen et al.. [12]. Jakobsen et al. detected strong absorption from singly ionized helium along the line of sight of quasar Q0302-003 at $z \simeq 3.3$. Their $90 \%$ upper bound on the continuum optical depth from singly ionized helium was $\tau_{\mathrm{GP}}^{\mathrm{He} I \mathrm{I}} \geq 1.7$. As there are only upper bounds on $\tau_{\mathrm{GP}}^{\mathrm{HI}}$ and $\tau_{\mathrm{GP}}^{\mathrm{HeI}}$, the presence of singly ionized helium in the IGM puts stringent constraints on the magnitude and spectrum of homogeneous sources of photoionization [13]. Radiatively decaying neutrinos is one such homogenous source of photoionization. In this scenario, a massive neutrino $\left(\nu_{h}\right)$ decays into a photon $(\gamma)$ and a light neutrino $\left(\nu_{\ell}\right)$ [14,15]. If $m_{\nu_{\ell}} \ll m_{\nu_{h}}$ - the case we consider-the photon and the light neutrino equally share the rest energy of $\nu_{h}$. We assume the heavy neutrino to be tau neutrino $\left(\nu_{\tau}\right)$, while the light neutrino can be either $\nu_{\mu}$ or $\nu_{e}$. To ionize singly ionized helium the mass of tau neutrino must be greater than $108.8 \mathrm{eV}$ (the ionization potential of singly ionized helium is $54.4 \mathrm{eV}$ ). The closure density constraint restricts the mass of a stable neutrino (or a neutrino which decays with a rate less than $t_{0}^{-1}, t_{0}$ being the present age of the universe) to be less than $91 \mathrm{~h}^{2} \mathrm{eV}$. This constraint forces the rate of neutrino decay to be greater than $t_{0}^{-1}$. As a radiative decay lifetime of less than $t_{0}$ is forbidden by several constraints, the neutrinos must predominantly decay into particles which are invisible (majoron, neutrinos etc.) with decay rate $\tau^{-1} \gg t_{0}^{-1}$, with a small branching ratio $B$ for the radiative mode (radiative lifetime $\left.\tau_{\gamma}=\tau / B\right)$.

In the presence of decaying neutrino, the thermal history of the universe can change considerably. First, as soon as the neutrinos become nonrelativistic the universe becomes matter dominated $\left(z=z_{1}\right)$. The universe remain matter dominated, with massive neutrinos dominating the energy density, until the time the neutrinos decay $\left(z=z_{d}\right)$ into relativistic decay products. (For simplicity we assume that all relativistic products of decaying neutrinos remain relativistic upto the present epoch.) Thus the epoch of neutrino decay is followed by a period of radiation domination in the universe. Depending on the mass and lifetime of decaying neutrinos and the energy density of other nonrelativistic matter $\left(\Omega_{N R}\right)$ in the universe, another period of matter domination could follow $\left(z=z_{2}\right)$. In the foregoing we have assumed the neutrinos to decay instantanously, which would result in a sudden change in matter and radiation domination. It is a good assumption for calculating the time-redshift relationship and for computing the age of the universe in the presence of decaying neutrinos [16]. In this paper we assume the universe to be spatially flat i.e., 
$\Omega_{0} \simeq \Omega_{\nu}+\Omega_{N R}=1$, where $\Omega_{N R}$ is the energy density due to all other non-relativistic matter.

Helium is the second most dominant primordial element ( $8 \%$ by number). In a uniform IGM, the proper density of helium is

$$
n_{\mathrm{He}}=6.8 \times 10^{-7} \Omega_{I G M} h^{2} \mathrm{~cm}^{-3} .
$$

Throughout this paper we take $\Omega_{I G M}=0.05$ and $h=1 / 2$. For a uniform IGM, any resonance line at wavelength $\lambda$ and oscillator strength $\mathrm{f}$, of singly ionized helium with a proper number density $n_{\mathrm{HeII}}(z)$, will produce an optical depth $[17,18]$

$$
\tau_{\mathrm{GP}}^{\mathrm{He} I \mathrm{I}}=\frac{\pi^{2}}{m_{e} c} f H^{-1}(z) \lambda n_{\mathrm{He} \text { II }}(z) .
$$

The dominant scattering is at wavelength $304 \AA$. Using this, $\tau_{\mathrm{GP}}^{\mathrm{He} I \mathrm{II}}>1.7$ at $z=3.3$ translates into a lower bound on the fraction of singly ionized helium, $y_{2}>1.2 \times 10^{-3}$, where $y_{2}=n_{\mathrm{HeII}} / n_{\mathrm{He}}$. Using that lower limit on $y_{2}$ is much less than unity, equilibrium between recombination and ionization processes can be assumed, which allows one to express $y_{2}$ as

$$
y_{2} \simeq 0.026 \times 10^{-10} \frac{(1+z)^{3}}{n_{\gamma}(z)} .
$$

Here

$$
n_{\gamma}(z)=\frac{\epsilon_{0}^{3}}{\pi^{2}} \int_{\epsilon_{0}}^{m_{\nu} / 2} \frac{d k}{k} f_{d}(k, t) \exp \left(-\tau\left(z, z_{e}, k\right) \quad \mathrm{cm}^{-3} ;\right.
$$

$\epsilon_{0}=54.4 \mathrm{eV}$ is the ionization potential of singly ionized helium; we have assumed the temperature of IGM $T=1.5 \times 10^{4} \mathrm{~K} ; f_{d}(k, t)$ is the distribution function of decay photons; $\tau\left(z, z_{e}, k\right)$ is the optical depth (to be described below) suffered by a photon which is emitted at $z_{e}$ and observed at $z$ with a wave number $k$. In the limit of small energy transfer between electrons and decay photons (a valid assumption for $z_{d} \lesssim 10^{4}$ ), the distribution function of photons can be written as $[1,8]$

$$
f_{d}(k, t)=\frac{B \pi^{2} n_{\nu}(\tau)}{n\left(m_{\nu} / 2\right)^{1 / n} k^{3-1 / n}}\left[\frac{\tau}{t}\right]^{3 n-1} \exp \left[-(t / \tau)\left(2 k / m_{\nu}\right)^{1 / n}\right] \Theta\left(m_{\nu} / 2-k\right) .
$$

Here $\mathrm{n}=1 / 2,2 / 3$ for radiation-dominated and matter-dominated epochs respectively.

Before discussing the results of our analysis, we briefly review some of the other bounds on radiatively decaying neutrinos. If neutrinos have a coupling to photons then, during a supernovae explosion, a photon flux coincidental with the neutrino flux from the supernovae should be observed. No such flux was observed during supernovae SN1987A. This has been used to constrain the parameter space of radiatively decaying neutrino [6]:

$$
\tau m_{\nu} \geq 2 \times 10^{19} B \mathrm{eV} \mathrm{sec},
$$

for neutrino masses between $100 \mathrm{eV}$ and a few $\mathrm{MeV}$. Other major astrophysical constraint comes from the study of red giant stars. The dominant energy loss mechanism in red giants is the decay of plasmons, formed in the core of the star. The rate of plasmon decay is enhanced if neutrinos have a magnetic moment. 
This can lead to precipitous cooling of the star, which is in conflict with known stellar evolution time scales. A study of such a process constrains the neutrino transition moment $\mu_{12} \lesssim 10^{-12} \mu_{B}$, which translates to [7]

$$
\tau \gtrsim 2.1 \times 10^{22} B \sec \left(\frac{m_{\nu}}{1 \mathrm{eV}}\right)^{-3}, \quad \text { for } m_{\nu} \leq 10 \mathrm{keV} .
$$

An interesting bound on trasition moment of radiatively decaying Dirac neutrinos can be obtained if the universe had a primordial magnetic field. The presence of magnetic field can cause the right-handed neutrino to be in theraml equilibrium with other relativistic particles in the universe at the time of nucleosynthesis, thereby adding an extra relativistic species, which is unaccetable. From such an analysis, a lower bound on trasition moment $\mu_{12} \leq 5 \times 10^{-14}\left(30 \mathrm{eV} / m_{\nu}\right)^{4 / 3} \mu_{B}$ can be obtained [19]; or in terms of neutrino lifetime

$$
\tau \geq 2.5 \times 10^{22}\left(\frac{30 \mathrm{eV}}{m_{\nu}}\right)^{4 / 3} \text { sec. }
$$

However, as there is no evidence of the existence of primordial magnetic field, this constraint may not apply.

Another stringent constraint on radiatively decaying neutrinos comes from the planckian nature of CBR. Photons emitted by decaying neutrinos can heat the electrons in the IGM; hot electrons can transfer this energy to CBR photons. If this process takes place at redshifts $\lesssim 10^{5}$, the spectrum of CBR is altered [20]. As CBR is known to be a planckian to a very high accuracy [21], the decay of neutrinos into photon mode is severly restricted $[8]$

$$
B\left(\frac{m_{\nu}}{1 \mathrm{eV}}\right)^{2} \lesssim 4.1 \times 10^{2}
$$

Finally, the decay photons constitute a diffuse background at wavelengths $\lesssim m_{\nu} / 2$. In recent years, rocket-borne experiments have placed upper bounds on diffuse, background photon flux in ultra-violet (UV), a wavelength range in which radiatively decaying neutrinos contribute most significantly [22]; in addition there exist several bounds on the extragalctic hydrogen-ionizing flux from astrophysical observations [23].

In this paper we point out that radiatively decaying neutrinos with masses above $108.8 \mathrm{eV}$ are subject to an additional constraint: the existence of singly ionized helium in the IGM. From eq.(3), it is clear that if $n_{\gamma}(3.3) \gtrsim 10^{-7}$, observed bound on $\tau_{\mathrm{GP}}^{\mathrm{HeII}}$ would be violated. To calculate $n_{\gamma}(z)$, sources of absorption must be identified. We consider two sources of absorption: diffuse IGM and lyman- $\alpha$ systems. Optical depth due to diffuse IGM is

$$
\tau_{\text {diffuse }}\left(z, z_{e}, k\right) \simeq \int_{z_{e}}^{z} d z\left|\frac{d t}{d z}\right| n_{\mathrm{He}} \sigma_{\mathrm{HeII}}(k) y_{2},
$$

where $\sigma_{\text {HeII }}(k)$ is the photoionization cross section from the ground state [24]. $\tau_{\text {cloud }}$, the avarage attenuation of photon flux due to poisson-distributed clouds, is given by

$$
\tau_{\text {cloud }}\left(z, z_{e}, k\right) \simeq \int_{z_{e}}^{z} \int_{0}^{\infty} d z d N_{\mathrm{HI}} \mathcal{P}\left(N_{\mathrm{HI}}, z\right)\left\{1-\exp \left[-N_{\mathrm{HeII}} \sigma_{\mathrm{HeII}}(k)\right]\right\}
$$


Here $N_{\mathrm{HI}}$ and $N_{\mathrm{HeII}}$ correspond to column densities of neutral hydrogen and singly ionized helium in the clouds and $\mathcal{P}\left(N_{\mathrm{HI}}, z\right)$ is the number density of clouds in a given column density and redshift interval. In ionization equilibrium, $N_{\mathrm{HeII}}$ can be inferred from known column densities of neutral hydrogen $N_{\mathrm{HI}}$

$$
N_{\mathrm{HeII}}=N_{\mathrm{HI}} \times \frac{n_{\gamma}^{\mathrm{HI}}}{n_{\gamma}^{\mathrm{HeII}}} \quad \mathrm{cm}^{-2}
$$

here $n_{\gamma}^{\mathrm{HI}}$ is the effective number of photons which ionize neutral hydrogen. From 'proximity effect' [25], $n_{\gamma}^{\mathrm{HI}}$ is known to approximately constant for $2 \lesssim z \lesssim 4$ with a lower limit $n_{\gamma}^{\mathrm{HI}} \gtrsim 6.2 \times 10^{-5} \mathrm{~cm}^{-3}$. For some of the paramter space for which $\tau_{\mathrm{GP}}^{\mathrm{He} I \mathrm{I}} \gtrsim 1$, the value of $n_{\gamma}^{\mathrm{HI}}$ from decaying neutrinos is less than the lower limit from 'proximity effect'. Hence we include the contribution from 'proximity effect' in $n_{\gamma}^{\mathrm{HI}}$. It is necessary to include this additional source of photons to correctly determine the optical depth (eq.(10)). For $\mathcal{P}(N, z)$, we take models A1 and A2 of Miralda-Escudé and Ostriker [18], in the redshift range $1 \leq z \leq 5$.

Our results are shown in figs. 1-4, along with other constraints. To gauge the effect of absorption, results in case of no absorption are also shown. In some range of masses, constraints on $B$ from the detection of singly ionized helium can be a few orders more stringent than any other constraint. Our results can be qualitatively understood. The photon flux at any energy $\mathrm{E}$ is determined by the the shape of decay photon spectrum (eq(5)). At any given redshift, if the exponent in photon spectrum can be neglected for photon energies $\gtrsim 54.4 \mathrm{eV}$ then photon flux at these energies

$$
\mathcal{F}(E) \propto E^{3} \times f_{d}(E) \simeq E^{3-1 / n}
$$

Thus photon spectrum is extremely hard (for comparsion, $\mathcal{F} \propto E^{-\alpha}, 0.7 \leq \alpha \leq 1.5$, for a photon background dominated by quasars). The bounds on $B$ are most stringent in this region. As $m_{\nu}$ is decreased for a given $\tau$, the exponent in decay photon spectrum begins to be important. This leads to a decrease in the number of photons above energy $54.4 \mathrm{eV}$ as well as to an increase in absorption (eq(10)), because the photon spectrum becomes soft for energies $\simeq 54.4 \mathrm{eV}$. And so larger values of $B$ are required to ionize the singly ionized helium. Our results are valid for $10^{12} \mathrm{sec} \leq \tau \leq 10^{16} \mathrm{sec}$. It is because for $\tau$ much less than $10^{12}$, the assumption that interaction between photons and electrons can be neglected in determining the spectrum of the decay photon breaks down. For $\tau \geq 10^{16} \mathrm{sec}$, neutrino masses above $108.8 \mathrm{eV}$ violate the closure density constraints. (All the region shown in figs (1)-(4) is compatible with closure density bounds.) The bounds on radiative lifetime can be translated into bounds on magnetic moment $\mu_{\nu}$ of radiatively decaying neutrinos. For Dirac neutrinos, radiative lifetime $\tau_{\gamma}(=\tau / B)$ is related to neutrino transition moment $\mu_{12}$ as [26]

$$
\tau_{\gamma}=10^{23}\left(\frac{30 \mathrm{eV}}{m_{\nu}}\right)^{3}\left(\frac{10^{-14} \mu_{B}}{\mu_{12}}\right)^{2} \text { sec. }
$$

The bounds on $\mu_{12}$ from the existence of singly ionized helium in the IGM can be summarized, for model A2 for absorption from lyman- $\alpha$ systems, as: 
(1) For $\tau>1.6 \times 10^{18} \sec \left(1 \mathrm{eV} / m_{\nu}^{2}\right)$,

$$
\mu_{12} \lesssim 4-8 \times 10^{-17} \mu_{B}
$$

in the mass range $110 \mathrm{eV} \lesssim m_{\nu} \lesssim 10 \mathrm{keV}$.

(2) For $1.6 \times 10^{18} \mathrm{sec}\left(1 \mathrm{eV} / m_{\nu}^{2}\right) \gtrsim \tau \lesssim 5 \times 10^{17} \mathrm{sec}\left(1 \mathrm{eV} / m_{\nu}^{2}\right)$,

$$
\mu_{12} \lesssim 8-100 \times 10^{-17} \mu_{B},
$$

in the mass range $110 \mathrm{eV} \lesssim m_{\nu} \lesssim 10 \mathrm{keV}$.

For $\tau \lesssim 5 \times 10^{17} \sec \left(1 \mathrm{eV} / m_{\nu}^{2}\right)$, other bounds become more significant (figs. 1-4).

Our bounds on $\mu_{12}$ are not restrictive enough to constrain the standard Wienberg-Salam model. However, several extensions of the standard model like the left-right symmetric model, charged-Higgs model, and broken R-parity supersymmetric model can give large transition moments (for a recent review see [27], [28]), and therefore would be constrained by the bounds we derive. As the most stringent constraints are obtained in the range $\tau \gtrsim 1.6 \times 10^{18} \sec \left(1 \mathrm{eV} / m_{\nu}^{2}\right)$, our results can be used to put an upper bound on $\tau$. It should be pointed out that the though these values of $\tau$ are not constrained by the closure density constraints, age and structure formation constraints may rule out much of the range of $\tau$ in which most stringent constraints on $\mu_{12}$ are obtained ([29], [30]).

We now discuss various sources of errors in our estimates from uncertainties in input parameters. (As GP tests are quite sensitive and model independent, our analysis is free from any errors of modelling.) First, it has been pointed out that a large contribution to Jakobsen et al.'s bound of $\tau_{\mathrm{GP}}^{\mathrm{HeII}} \geq 1.7$ might come from line blankating [13]. But the presence of a foreground quasar along the line of sight to Q302-003 might reduce the optical depth due to line blankating quite significantly [31]. In any case, these uncertainties should not change bounds on $B$ by more than an order. (It should however be pointed out that even in case most of the contribution to optical depth observed by Jakobsen et al. comes from line blankating, equally stringent bounds on radiatively decaying neutrinos can be obtained. If line blankating causes most of the optical depth, one requires $S_{L} \equiv n_{\gamma}^{\mathrm{HI}} / n_{\gamma}^{\text {HeII }} \gtrsim 65$. This puts strong constraints on the spectrum of radiatively decaying neutrinos.) Other input parameters like $\Omega_{I G M}, T$, and $h$ can change our estimate by a factor of few. Another source of uncertainty lies in the choice of a model for absorption from lyman- $\alpha$ system. From observations, Model A2 of Miralda-Escude and Ostriker gives a fair picture of absorption due to lyman- $\alpha$ systems at high redshifts. However, Meiksin \& Madau [32] have argued that absorption due to lyman- $\alpha$ clouds can be smaller (as compared to model A2 of Miralda-Escude and Ostriker). On the other hand, Lanzetta [33] has reported a rapid evolution of lyman-limit systems at high redshifts, which would increase the absorption. However these uncertainties in absorption from lyman- $\alpha$ systems do not change values calculated using model A2 by more than a factor of few.

To conclude, we showed that the presence of singly ionized helium in the diffuse IGM can put extremely 
stringent constraints on radiatively decaying neutrinos. In particular, for $\tau \gtrsim a$ few $\times 10^{18} \sec \left(1 \mathrm{eV} / m_{\nu}^{2}\right)$, the bounds on radiative lifetime (or equivalently on transtion moment $\mu_{12}$ ) can be several orders more stringent than the previously known astrophysical and cosmological constraints. More recently, large optical depth from singly ionized helium has been detected in the spectra of two more quasars [34], which would allow one to obtain similar constraints on radiatively decaying neutrinos. Our results also show that the presence of primordial elements in the IGM generically give very restrictive bounds on radiatively decaying neutrinos.

\section{Acknowledgements}

The auther would like to thank A. Goyal, B. Nath, T. Padmanabhan, and P. Pal for useful discussions. 


\section{References}

[1] A. De Rajula, S. L. Glashow, Phys. Rev. Lett. 45, 942 (1980).

[2] D. W. Sciama, Phys. Rev. Lett. 65, 2839 (1990).

[3] D. W. Sciama, Modern Cosmology and the Dark Matter Problem (Cambridge University Press, 1993).

[4] E. W. Kolb and M. S. Turner, The Early Universe (Addison-Wesley, Redwood City, CA, 1990).

[5] R. Cowsik, Phys. Rev. Lett. 39, 784 (1977).

[6] E. W. Kolb and M. S. Turner, Phys. Rev. Lett. 62, 509 (1989); S. A. Bludman, Phys. Rev. D 45, 4720 (1992); L. Oberaurer et al., Astropar. Phys. 1, 377 (1993).

[7] G. G. Raffelt, Astrophys. J. 365, 559 (1990);V. Castellani and Dengl'Innocenti, Astrophys. J. 402, 574 (1993).

[8] J. Bernstein and S. Dodelson, Phys. Rev. D 41, 354 (1990); S. Dodelson and J. M. Jubas, MNRAS 266, 886 (1994).

[9] J. E. Gunn and B. A. Peterson, Astrophys. J. 142, 1633 (1965).

[10] E. B. Jenkins and J. P. Ostriker, Astrophys. J. 376, 33 (1991); E. Giallongo et al., Astrophys. J. 398, L9 (1992); J. K. Webb, MNRAS 255, 319 (1991).

[11] T. M. Tripp, R. F. Green, and J. Bechtold, Astrophys. J. 364, L29 (1990); E. A. Beaver et al., Astrophys. J. 337, L1 (1991); D. Reimers et al., Nature (London) 360, 561 (1992).

[12] P. Jakobsen et al., Nature (London), 370, 35 (1994).

[13] P. Madau and A. Meiksin, Astrophys. J. 433, L53 (1994); A. Songaila, E. L. Hu, and L. L. Cowie, Nature (London) 375, 124 (1995).

[14] M. Fukugita, Phys. Rev. Lett. 61, 1046 (1988).

[15] R. Cowsik and J. McClelland, Phys. Rev. Lett. 29, 669 (1972).

[16] M. S. Turner, Phys. Rev. D 31, 1212 (1985).

[17] T. Padmanabhan, Structure Formation in the Universe (Cambridge University Press, 1993).

[18] J. Miralda-Escudé and J. P. Ostriker, Astrophys. J. 350, 1 (1990); J. Miralda-Escudé, MNRAS 262, 273 (1993).

[19] K. Enqvist, P. Olesen and V. Seminov, Phys. Rev. Lett. 69, 2157 (1992).

[20] Y. B. Zeldovich and R. A. Sunyaev, Asron. Zh. 46, 775 (1969)

[21] J. C. Mather et al., Astrophys. J. 420, 439 (1994).

[22] S. Bowyer, ARA \& A, 29, 59 (1991); R. C. Henry, ARA \& A, 29, 89 (1991).

[23] R. J. Reynolds et al., Astrophys. J. 309, L9 (1986); P. Maloney, Astrophys. J. 414, 41 (1993).

[24] R. L. Brown, Astrophys. J. 164, 387 (1971); A. A. Zdziarski and R. Svensson, Astrophys. J. 344, 551 (1989).

[25] S. Bajtik, R. C. Duncan, and J. P. Ostriker, Astrophys. J. 327, 570 (1988).

[26] W. Marciano and A. I. Sanda, Phys. Lett. B 67, 303, (1977).

[27] R. N. Mohapatra and P. B. Pal, Massive Neutrinos in Physics and Astrophysics (World Scientific, 
Singapore, 1991).

[28] M. Fukugita and T. Yanagida, in Physics and Astrophysics of Neutrinos eds. M. Fukugita and A. Suzuki (Springer-Verlag, 1994).

[29] S. K. Sethi, in preparation.

[30] M. White, G. Gelmini, and J. Silk, Phys. Rev. D 51, 2669(1995)

[31] B. B. Nath and S. K. Sethi, submitted for publication.

[32] A. Meiksin and P. Madau, Astrophys. J. 412, 34 (1993).

[33] K. M. Lanzetta, Astrophys. J. 375, 1 (1991).

[34] D. Tytler, 1994, unpublished; A. Davidsen, 1995, unpublished. 


\section{Figure Captions}

Figure 1 : For $\tau=10^{12} \mathrm{sec}$, constraints from singly ionized helium (solid lines marked with chosen model of absorption from lyman- $\alpha$ systems) are plotted with constraints from supernova (dashed line), CBR spectrum (dotted line), UV background for absorption model A2 (dot-dot-dot-dashed line), and primordial magentic field (dot-dashed line). Region above the curves is ruled out by the respective constraints.

Figure 2 : Same as fig. (1) but for $\tau=10^{13} \mathrm{sec}$.

Figure 3 : Same as fig. (1) but for $\tau=10^{14} \mathrm{sec}$.

Figure 4 : Same as fig. (1) but for $\tau=10^{15}$ sec. 


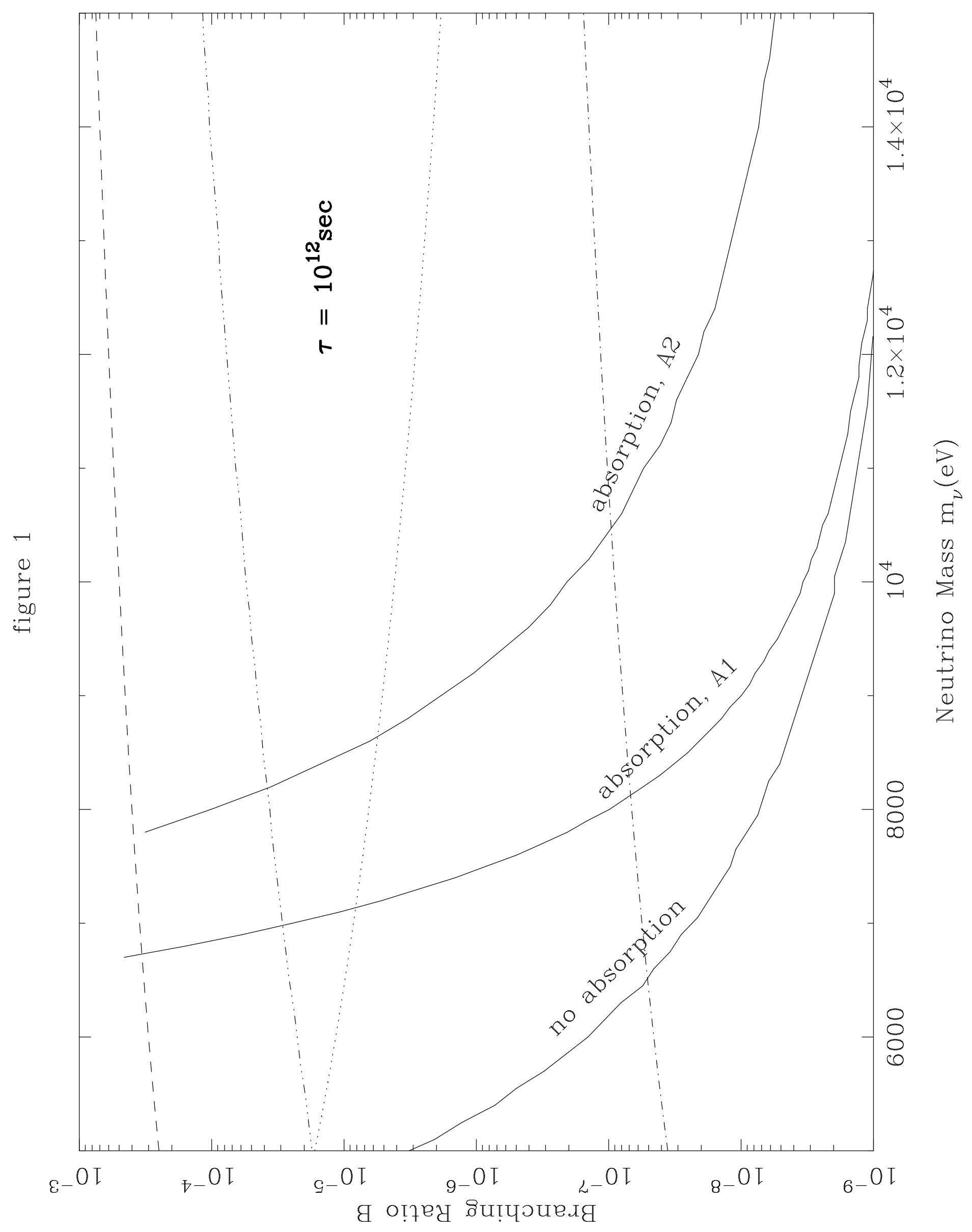




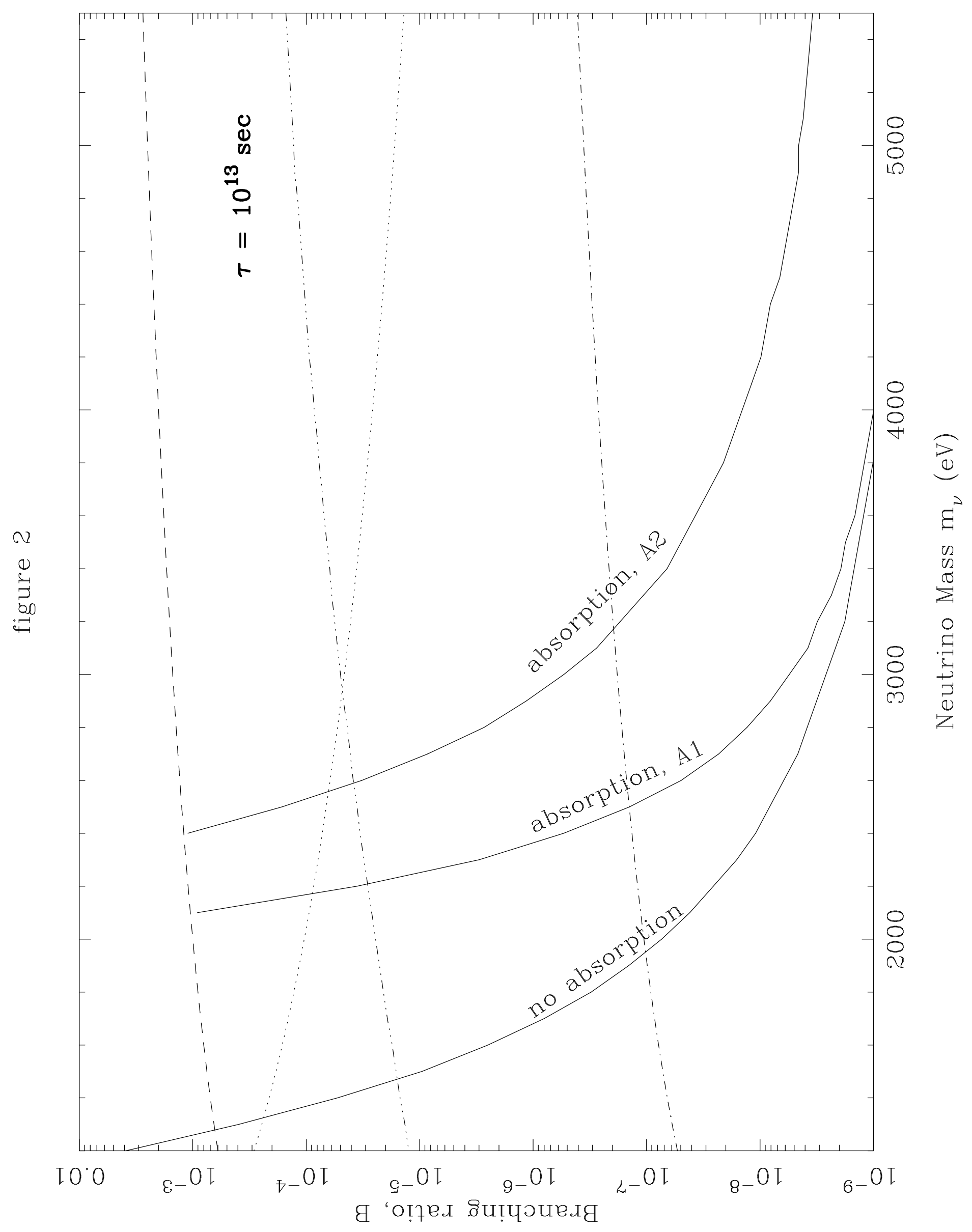




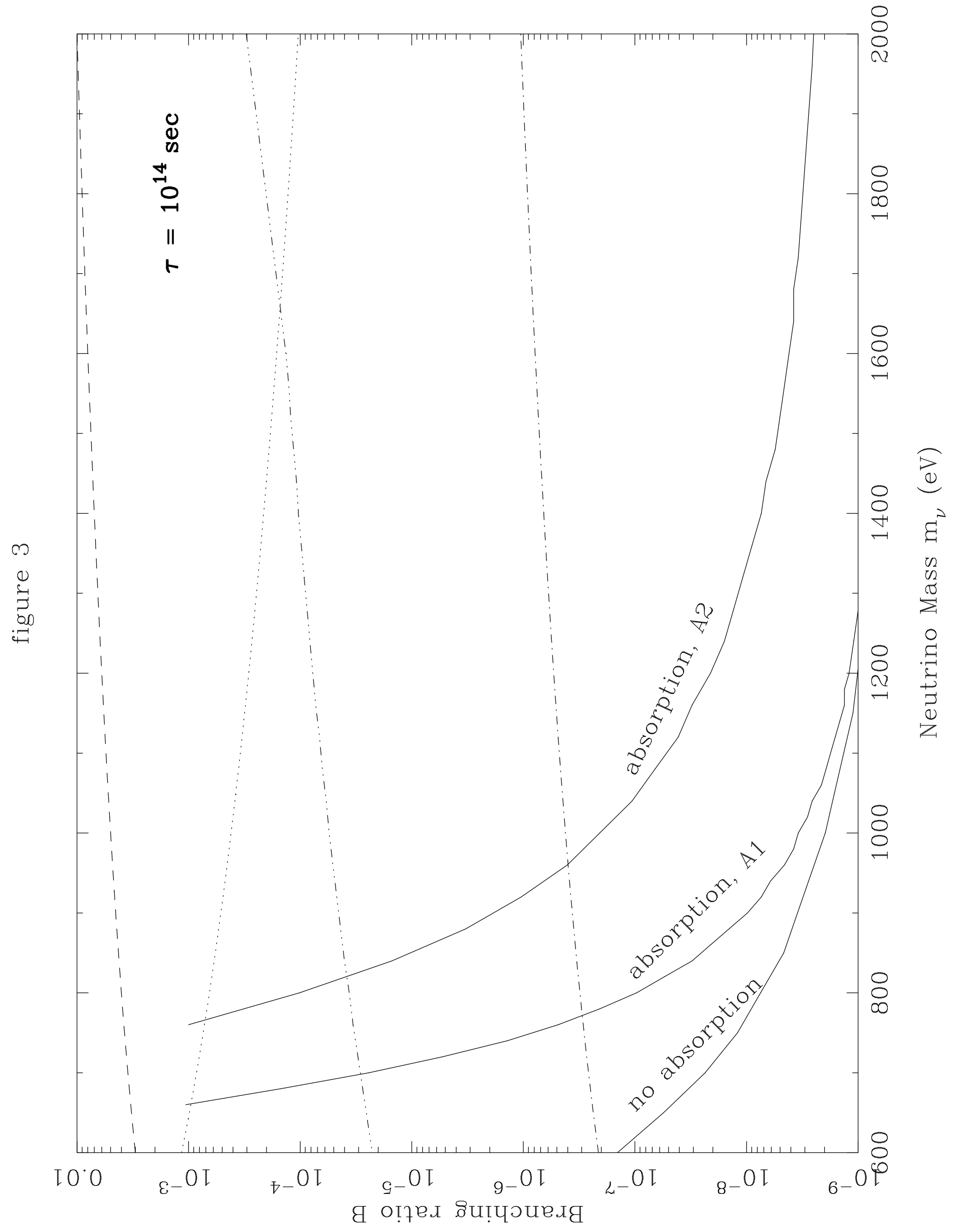




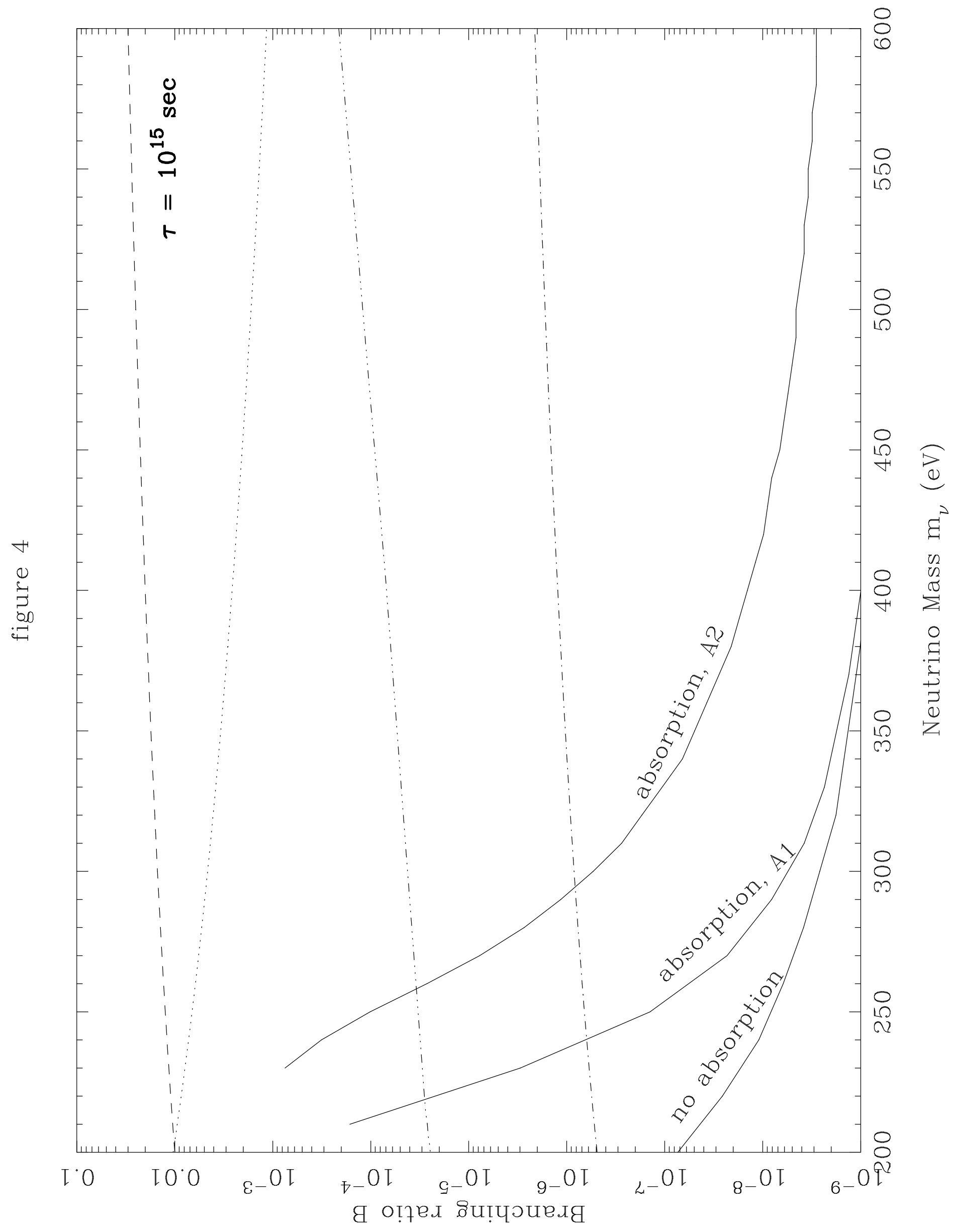

